\title{
Infinitely Many Standing Waves for the Nonlinear Chern-Simons-Schrödinger Equations
}

\author{
Jinmyoung Seok \\ Department of Mathematics, Kyonggi University, 154-42 Gwanggyosan-ro, Yeongtong-gu, Suwon 443-760, Republic of Korea \\ Correspondence should be addressed to Jinmyoung Seok; jmseok512@gmail.com
}

Received 2 July 2015; Accepted 5 August 2015

Academic Editor: Andrei D. Mironov

Copyright (C) 2015 Jinmyoung Seok. This is an open access article distributed under the Creative Commons Attribution License, which permits unrestricted use, distribution, and reproduction in any medium, provided the original work is properly cited.

We prove the existence of infinitely many solutions of the nonlinear Chern-Simons-Schrödinger equations under a wide class of nonlinearities. This class includes the standard power-type nonlinearity with exponent $p>4$. This extends the previous result which covers the exponent $p>6$.

\section{Introduction}

In $[1,2]$, Jackiw and Pi introduce a nonrelativistic model that the nonlinear Schrödinger dynamics is coupled with the Chern-Simons gauge terms as follows:

$$
\begin{aligned}
i D_{0} \phi+\left(D_{1} D_{1}+D_{2} D_{2}\right) \phi & =-\lambda|\phi|^{p-2} \phi, \\
\partial_{0} A_{1}-\partial_{1} A_{0} & =-\operatorname{Im}\left(\bar{\phi} D_{2} \phi\right), \\
\partial_{0} A_{2}-\partial_{2} A_{0} & =\operatorname{Im}\left(\bar{\phi} D_{1} \phi\right), \\
\partial_{1} A_{2}-\partial_{2} A_{1} & =-\frac{1}{2|\phi|^{2}} .
\end{aligned}
$$

Here, $i$ denotes the imaginary unit, $\partial_{0}=\partial / \partial t, \partial_{1}=\partial / \partial x_{1}$, $\partial_{2}=\partial / \partial x_{2}$ for $\left(t, x_{1}, x_{2}\right) \in \mathbb{R}^{1+2}, \phi: \mathbb{R}^{1+2} \rightarrow \mathbb{C}$ is a complex scalar field, $A_{\mu}: \mathbb{R}^{1+2} \rightarrow \mathbb{R}$ is a component of gauge potential and $D_{\mu}=\partial_{\mu}+i A_{\mu}$ is a covariant derivative for $\mu$ running over $0,1,2$, and $\lambda>0$ is a parameter. The Chern-Simons gauge theory appears in the 1980s to explain electromagnetic phenomena of anyon physics such as the high temperature superconductivity or the fractional quantum Hall effect. In this paper, we are interested in standing wave solutions of (1). In [3], the authors introduce a standing wave ansatz of the following form:

$$
\begin{aligned}
\phi(t, x) & =u(|x|) e^{i \omega t}, \\
A_{0}(t, x) & =k(|x|),
\end{aligned}
$$

$$
\begin{aligned}
& A_{1}(t, x)=\frac{x_{2}}{|x|^{2}} h(|x|), \\
& A_{2}(t, x)=-\frac{x_{1}}{|x|^{2}} h(|x|),
\end{aligned}
$$

where $\omega>0$ is a phase frequency and $u, k, h$ are real valued functions on $[0, \infty)$ such that $h(0)=0$. Inserting (2) into (1), one may check from direct computation that (1) is reduced to the following nonlinear nonlocal elliptic equation:

$$
\begin{aligned}
& \Delta u- \omega u-\left(\int_{|x|}^{\infty} \frac{h_{u}(s)}{s} u^{2}(s) d s\right) u-\frac{h_{u}^{2}(|x|)}{|x|^{2}} u \\
&+\lambda|u|^{p-2} u=0 \quad \text { in } \mathbb{R}^{2},
\end{aligned}
$$

where $h_{u}(s)=\int_{0}^{s}(1 / 2) u^{2}(\sigma) d \sigma$. See [3] for its derivation. It is shown in [3] that (3) is an Euler-Lagrange equation of a $C^{1}$ functional,

$$
\begin{aligned}
J(u):= & \frac{1}{2} \int_{\mathbb{R}^{2}}|\nabla u|^{2}+\omega u^{2}+\frac{h_{u}^{2}(|x|)}{|x|^{2}} u^{2} d x \\
& -\frac{1}{p} \int_{\mathbb{R}^{2}}|u|^{p} d x \quad \text { on } H_{r}^{1}\left(\mathbb{R}^{2}\right),
\end{aligned}
$$

where $H_{r}^{1}\left(\mathbb{R}^{2}\right)$ denotes the set of radially symmetric functions in standard Sobolev space $H^{1}\left(\mathbb{R}^{2}\right)$. Investigating the structure 
of $J$, the authors of [3] obtain several existence and nonexistence results for (3), depending on the range of $p>2$ and $\lambda>0$. Recently, Pomponio and Ruiz [4] improve the results in [3] for the case $p \in(2,4)$. They find a threshold for the behavior of $J$, depending on $\omega>0$. They also study (3) on bounded domain in [5].

In this paper, we are concerned with the existence of infinitely many solutions of (3). It is proved in [6] that if $p>6, J$ enjoys the symmetric mountain pass geometry and satisfies the (PS) condition so that the well-known symmetric mountain pass lemma (see [7]) applies to show there exist infinitely many critical points of $J$. For $p \in(4,6]$, it turns out that $J$ still enjoys the symmetric mountain pass geometry although checking the (PS) condition is not easy job. One aim of this paper is to show nevertheless $J$ still admits infinitely many critical points for $p>4$. Moreover, we will replace the power-type nonlinearity $\lambda|u|^{p-2} u$ of (3) with more general one as follows:

$$
\begin{aligned}
\Delta u- & \omega u-\left(\int_{|x|}^{\infty} \frac{h_{u}(s)}{s} u^{2}(s) d s\right) u-\frac{h_{u}^{2}(|x|)}{|x|^{2}} u \\
& +V\left(u^{2}\right) u=0 .
\end{aligned}
$$

The structure conditions for $V$ are given by the following:

(V1) Consider $V \in C^{1}\left(\mathbb{R}_{+}, R\right)$ such that $V(0)=V^{\prime}(0)=0$.

(V2) Consider $\limsup _{s \rightarrow \infty}\left|V^{\prime}(s) / s^{p-1}\right|<\infty$, $\limsup _{s \rightarrow \infty}\left|V(s) / s^{p}\right|<\infty$ for some $p>1$.

(V3) There exists some $\alpha>1$ such that $\alpha V^{\prime}(s) / s^{2-1 / \alpha}-$ $V(s) / s^{3-1 / \alpha}$ is monotonically increasing to $\infty$ as $s \rightarrow$ $\infty$.

Observe that assumptions (V1)-(V3) include the power-type nonlinearity $(\lambda / p)|u|^{p}, p>4$.

Theorem 1. Assume (V1)-(V3). Then, (5) admits infinitely many solutions.

We refer to the work of Cunha et al. [8] that if we insert a sufficiently small parameter $q>0$ into (5) as in

$$
\begin{aligned}
\Delta u- & \omega u-q\left(\int_{|x|}^{\infty} \frac{h_{u}(s)}{s} u^{2}(s) d s\right) u-q \frac{h_{u}^{2}(|x|)}{|x|^{2}} u \\
& +V\left(u^{2}\right) u=0,
\end{aligned}
$$

then much more general assumptions for $V$, the so-called Berestycki-Lions conditions [9], are sufficient for guaranteeing the existence and multiplicity of solutions of (6). In our work, we assume further than the Berestycki-Lions conditions but we do not need small parameter $q>0$. See also [10] in which Tan and Wan consider asymptotically linear nonlinearities.

To prove Theorem 1, we will apply the method employed in author's former paper [11] in which the SchrödingerPoisson equation, another nonlocal field equation similar to (5), is dealt with. Instead of generating (PS) sequences, we will show the existence and compactness of the socalled approximate solution sequences of $J$ which may be considered as more refined version of (PS) sequences. In Section 2, we give a definition of the approximate solution sequences of $J$. Some auxiliary lemmas are also prepared in Section 2. In Section 3, we prove the compactness of approximate solution sequences. In Section 4, we construct infinitely many approximate solution sequences whose energy levels go to infinity and complete the proof of Theorem 1 .

\section{Mathematical Settings and Preliminaries}

Let $H_{r}^{1}\left(\mathbb{R}^{2}\right)$ be the completion of $D:=\left\{u \in C_{c}^{\infty}\left(\mathbb{R}^{2}\right) \mid u(x)=\right.$ $u(|x|)\}$ with respect to the norm

$$
\|u\|=\left(\int_{\mathbb{R}^{2}}|\nabla u|^{2}+\omega u^{2} d x\right)^{1 / 2} .
$$

The dual space of $H_{r}^{1}\left(\mathbb{R}^{2}\right)$ is denoted by $H_{r}^{1}\left(\mathbb{R}^{2}\right)^{*}$. Arguing similarly to [3], it is easy to show (5) is an Euler-Lagrange equation of the $C^{1}$ functional

$$
\begin{array}{r}
J(u):=\frac{1}{2} \int_{\mathbb{R}^{2}}|\nabla u|^{2}+\omega u^{2}+\frac{h_{u}^{2}(|x|)}{|x|^{2}} u^{2}-V\left(u^{2}\right) d x \\
\text { on } H_{r}^{1}\left(\mathbb{R}^{2}\right) .
\end{array}
$$

In this paper, we search for infinitely many critical points of $J$ to prove Theorem 1 . To do this, we insert parameter $\lambda$ into $J$ as follows:

$$
\begin{aligned}
J_{\lambda}(u):= & \frac{1}{2} \int_{\mathbb{R}^{2}}|\nabla u|^{2}+\omega u^{2}+\frac{h_{u}^{2}(|x|)}{|x|^{2}} u^{2} \\
& -\lambda V\left(u^{2}\right) d x .
\end{aligned}
$$

Here $\lambda$ ranges over $[1 / 2,1]$. For a sequence $\left\{\lambda_{j}\right\} \in[1 / 2,1]$ which converges to 1 as $j \rightarrow \infty$, we say $\left\{\lambda_{j}, u_{j}\right\}$ is an approximate solution sequence of $J$ if $J_{\lambda_{j}}^{\prime}\left(u_{j}\right)=0$ for all $j$. In the following subsection, we state a variant of the famous Struwe's monotonicity trick [12], which plays a crucial role in constructing approximate solution sequences.

2.1. A Variant of Struwe's Monotonicity Trick. Let $X$ be Banach space. We say a subset $A \subset X$ is symmetric if $-v \in A$ for every $v \in A$. Let $A$ be a compact subset of $X$ and $B$ a closed subset of $A$. We denote by $\Gamma$ the set of every continuous odd function $\gamma: A \rightarrow X$ such that $\gamma(v)=v$ on $B$. Let $I$ be a closed interval in $\mathbb{R}$ and $J_{\lambda}$ one parameter family of even $C^{1}$ functional on $X$. We define a minimax level by

$$
c(\lambda):=\min _{\gamma \in \Gamma} \max _{v \in A} J_{\lambda}(\gamma(v)) .
$$

The following theorem is a variant of so-called Struwe's monotonicity trick [12]. A more general version of it is given in [11]. The property $(H)$ below is first proposed by Jeanjean and Toland in [13].

Theorem 2 (see [11]). Suppose that, for all $\lambda \in I$,

$$
c(\lambda)>\max _{v \in B} J_{\lambda}(v) .
$$


Then, for almost every $\lambda \in I$, there exists a norm-bounded (PS) sequence of $J_{\lambda}$ at level $c(\lambda)$, provided the following property $(H)$ for $J_{\lambda}$ holds:

(H) For given $\lambda_{0} \in I$, let $\left\{\lambda_{j}\right\} \in I$ be a sequence strictly increasing to $\lambda_{0}$ and $\left\{v_{j}\right\}$ a sequence in $X$ such that

$$
\begin{gathered}
-J_{\lambda_{0}}\left(v_{j}\right), \\
J_{\lambda_{j}}\left(v_{j}\right), \\
\frac{J_{\lambda_{j}}\left(v_{j}\right)-J_{\lambda_{0}}\left(v_{j}\right)}{\lambda_{0}-\lambda_{j}}
\end{gathered}
$$

are all uniformly bounded above for $j$. Then the following holds:

(i) $\left\{v_{j}\right\}$ is norm-bounded in $X$.

(ii) For given $\varepsilon>0$, there exists $N>0$ such that

$$
J_{\lambda_{0}}\left(v_{j}\right) \leq J_{\lambda_{j}}\left(v_{j}\right)+\varepsilon \quad \forall j \geq N
$$

2.2. Some Auxiliary Lemmas. Here, we prepare some lemmas which will be necessarily used for proving the main result. Define

$$
\widehat{J}_{\lambda}(u):=\frac{1}{2} \int_{\mathbb{R}^{2}} \frac{h_{u}^{2}(|x|)}{|x|^{2}} u^{2}-\lambda V\left(u^{2}\right) d x
$$

Lemma 3 (Lemma 3.2 in [3]). Let $\left\{u_{j}\right\} \in H_{r}^{1}\left(\mathbb{R}^{2}\right)$ be a sequence weakly converging to some $u_{0}$ in $H_{r}^{1}\left(\mathbb{R}^{2}\right)$ as $j \rightarrow \infty$. Then, for each $\varphi \in H_{r}^{1}\left(\mathbb{R}^{2}\right)$, it holds that $\widehat{J}_{\lambda}\left(u_{n}\right) \rightarrow \widehat{J}_{\lambda}\left(u_{0}\right)$, $\widehat{J}_{\lambda}^{\prime}\left(u_{n}\right) \varphi \rightarrow \widehat{J}_{\lambda}^{\prime}\left(u_{0}\right) \varphi$, and $\widehat{J}_{\lambda}^{\prime}\left(u_{n}\right) u_{n} \rightarrow \widehat{J}_{\lambda}^{\prime}\left(u_{0}\right) u_{0}$ as $j \rightarrow \infty$, up to a subsequence.
Lemma 4 (Pohozaev identity). Let $u \in H_{r}^{1}\left(\mathbb{R}^{2}\right)$ be a critical point of $J_{\lambda}$. Then one has

$$
\begin{aligned}
& \int_{\mathbb{R}^{2}} \omega u^{2} d x+2 \int_{\mathbb{R}^{2}} \frac{h_{u}^{2}(|x|)}{|x|^{2}} u^{2} d x-\int_{\mathbb{R}^{2}} \lambda V\left(u^{2}\right) d x \\
& \quad=0 .
\end{aligned}
$$

For each $u \in H_{r}^{1}\left(\mathbb{R}^{2}\right)$ and $t>0$, we define one parameter family of functions $u_{t} \in H_{r}^{1}\left(\mathbb{R}^{2}\right)$ by

$$
u_{t}(x):=t^{\alpha} u(t x) \text {. }
$$

For fixed $t>0$, we define a map $L_{t}: H^{1}\left(\mathbb{R}^{2}\right) \rightarrow H^{1}\left(\mathbb{R}^{2}\right)$ by $L_{t}(u)=u_{t}$. It is easy to see that $L_{t}$ is a continuous and linear map with the inverse $L_{1 / t}$. Thus $L_{t}$ is a linear isomorphism.

For each $u \in H^{1}\left(\mathbb{R}^{2}\right)$ and $\lambda \in[1 / 2,1]$, let $c_{u, \lambda}:[0, \infty) \rightarrow$ $\mathbb{R}$ be a function defined by

$$
c_{u, \lambda}(t):=J_{\lambda}\left(u_{t}\right)
$$

Lemma 5. For any $u \in H_{r}^{1}\left(\mathbb{R}^{2}\right)$ and $\lambda \in[1 / 2,1], c_{u, \lambda}$ admits a unique critical point $t_{0}>0$ on $(0, \infty)$; that is, $c_{u, \lambda}^{\prime}\left(t_{0}\right)=0$, such that $c_{u, \lambda}(t)$ is increasing on $\left(0, t_{0}\right)$, attains its maximum at $t_{0}$, and is decreasing to $-\infty$ on $\left(t_{0}, \infty\right)$.

Proof. By the change of variable, one can compute

$$
\begin{aligned}
& c_{u, \lambda}(t)=J_{\lambda}\left(t^{\alpha} u(t \cdot)\right)=\frac{1}{2}\left(t^{2 \alpha} \int_{\mathbb{R}^{2}}|\nabla u|^{2} d x\right. \\
& +t^{2 \alpha-2} \int_{\mathbb{R}^{2}} \omega u^{2} d x+t^{6 \alpha-4} \int_{\mathbb{R}^{2}} \frac{h_{u}^{2}(|x|)}{|x|^{2}} u^{2} d x \\
& \left.-t^{-2} \int_{\mathbb{R}^{2}} \lambda V\left(t^{2 \alpha} u^{2}\right) d x\right) .
\end{aligned}
$$

We differentiate it with respect to $t$ to get

$$
\begin{aligned}
& c_{u, \lambda}^{\prime}(t)=\alpha t^{2 \alpha-1} \int_{\mathbb{R}^{2}}|\nabla u|^{2} d x+(\alpha-1) t^{2 \alpha-3} \int_{\mathbb{R}^{2}} \omega u^{2} d x+(3 \alpha-2) t^{6 \alpha-5} \int_{\mathbb{R}^{2}} \frac{h_{u}^{2}(|x|)}{|x|^{2}} u^{2} d x+t^{-3} \int_{\mathbb{R}^{2}} \lambda V\left(t^{2 \alpha} u^{2}\right) d x \\
& -t^{-2} \int_{\mathbb{R}^{2}} \lambda V^{\prime}\left(t^{2 \alpha} u^{2}\right) \alpha t^{2 \alpha-1} u^{2} d x=t^{6 \alpha-5}(\underbrace{\frac{\alpha}{t^{4 \alpha-4}} \int_{\mathbb{R}^{2}}|\nabla u|^{2} d x+\frac{\alpha-1}{t^{4 \alpha-2}} \int_{\mathbb{R}^{2}} \omega u^{2} d x+(3 \alpha-2) \int_{\mathbb{R}^{2}} \frac{h_{u}^{2}(|x|)}{|x|^{2}} u^{2} d x}_{A(t)} \\
& -\underbrace{\int_{\mathbb{R}^{2}} \lambda\left[\frac{\alpha V^{\prime}\left(\left(t^{\alpha} u\right)^{2}\right)}{\left(\left(t^{\alpha} u\right)^{2}\right)^{2-1 / \alpha}}-\frac{V\left(\left(t^{\alpha} u\right)^{2}\right)}{\left(\left(t^{\alpha} u\right)^{2}\right)^{3-1 / \alpha}}\right]\left(u^{2}\right)^{3-1 / \alpha} d x}_{B(\mathrm{t})}) .
\end{aligned}
$$


Observe from assumption (V3) that $A(t)$ is strictly monotonically decreasing from infinity to the positive number $(3 \alpha-$ 2) $\int_{\mathbb{R}^{2}}\left(h_{u}^{2}(|x|) /|x|^{2}\right) u^{2} d x$ on $(0, \infty)$ and $B(t)$ is monotonically increasing to infinity on $(0, \infty)$. Therefore there is $t_{0}>0$ such that $A(t)>B(t)$ on $\left(0, t_{0}\right), A\left(t_{0}\right)=B\left(t_{0}\right)$, and $A(t)<B(t)$ on $\left(0, t_{0}\right)$. Also from assumption (V3), we deduce $B(t) \rightarrow \infty$ as $t \rightarrow \infty$. This proves the proposition.

We define a function $T: H^{1}\left(\mathbb{R}^{2}\right) \rightarrow \mathbb{R}$ by assigning a positive number $t$ satisfying $\left\|t^{-\alpha} u\left(t^{-1} \cdot\right)\right\|=1$ for any nonzero $u \in H_{r}^{1}\left(\mathbb{R}^{2}\right)$. The value $T(0)$ is defined by 0 .

Lemma 6. The function $T$ is well-defined and continuous even map on $[0, \infty)$.

Proof. To show the well-definedness of $T$, we have to show that there exists unique $t>0$ satisfying $\left\|t^{-\alpha} u\left(t^{-1} \cdot\right)\right\|=1$ for given nonzero $u \in H_{r}^{1}\left(\mathbb{R}^{2}\right)$. We note that this is equivalent to prove there is a unique solution $t>0$ of the equation

$$
g(t):=t^{2 \alpha}-\left(\int_{\mathbb{R}^{2}} \omega u^{2} d x\right) t^{2}-\int_{\mathbb{R}^{2}}|\nabla u|^{2} d x=0
$$

Arguing similarly to the proof of Lemma 5, we are able to see that $g(t)$ is monotonically decreasing on $\left(0, t_{0}\right)$ for some $t_{0}>0$, attains its unique local minimum at $t_{0}$, and is monotonically increasing to infinity on $\left(t_{0}, \infty\right)$. Therefore there is a unique positive zero of $g(t)$ since $g(0)=0$. Also, the implicit function theorem says that $T(u)$ is continuous on $[0, \infty)$ because $g^{\prime}(T(u)) \neq 0$. The evenness of $T$ follows from the fact that each coefficient of $(20)$ is even. This completes the proof.

\section{Compactness of Approximate Solution Sequences}

In this section, we prove the compactness of an approximate solution sequence $\left\{u_{j}\right\}$ of $J_{\lambda_{j}}$ when its energy $\left\{J_{\lambda_{j}}\left(u_{j}\right)\right\}$ is bounded above.
Proposition 7. Let $\lambda_{j} \in[1 / 2,1]$ be such that $\lambda_{j} \rightarrow 1$ as $j \rightarrow \infty$. Let $\left\{u_{j}\right\} \in H_{r}^{1}\left(\mathbb{R}^{2}\right)$ be a sequence of critical points of $J_{\lambda_{j}}$; that is, $J_{\lambda_{j}}^{\prime}\left(u_{j}\right)=0$. Suppose that $J_{\lambda_{j}}\left(u_{j}\right)<C$ for some $C>0$, independent of $j$. Then $u_{j} \rightarrow u_{0}$ in $H^{1}\left(\mathbb{R}^{2}\right)$ for some critical point $u_{0} \in H_{r}^{1}\left(\mathbb{R}^{2}\right)$ of $J\left(=J_{1}\right)$ up to a subsequence.

Proof. We divide the proof into two steps.

Step 1 (boundedness of $u_{j}$ ). We first prove that $\left\{u_{j}\right\}$ is bounded in $H_{r}^{1}\left(\mathbb{R}^{2}\right)$. Arguing indirectly, suppose that $\left\{u_{j}\right\}$ is unbounded. Let $T_{j}:=T\left(u_{j}\right)$, where the function $T$ is defined in Section 2. Equation (20) says $T_{j}$ is unbounded. Let $v_{j}(x):=$ $T_{j}^{-\alpha} u_{j}\left(T_{j}^{-1} x\right)$ so that $\left\|v_{j}\right\|=1$. Then, up to a subsequence, $\left\{v_{j}\right\}$ converges weakly in $H_{r}^{1}\left(\mathbb{R}^{2}\right)$ and strongly in $L^{q}\left(\mathbb{R}^{2}\right)$ for all $q>2$ to some $v_{0} \in H_{r}^{1}\left(\mathbb{R}^{2}\right)$. Since $u_{j}$ is a critical point of $J_{\lambda_{j}}$, we see that

$$
\begin{gathered}
\int_{\mathbb{R}^{2}}\left|\nabla u_{j}\right|^{2} d x+\int_{\mathbb{R}^{2}} \omega u_{j}^{2} d x+3 \int_{\mathbb{R}^{2}} \frac{h_{u_{j}}^{2}(|x|)}{|x|^{2}} u_{j}^{2} d x \\
-\int_{\mathbb{R}^{2}} \lambda_{j} V^{\prime}\left(u_{j}^{2}\right) u_{j}^{2} d x=J_{\lambda_{j}}^{\prime}\left(u_{j}\right) u_{j}=0 .
\end{gathered}
$$

Combining this with the Pohozaev identity (15), we obtain

$$
\begin{aligned}
& \alpha \int_{\mathbb{R}^{2}}\left|\nabla u_{j}\right|^{2} d x+(\alpha-1) \int_{\mathbb{R}^{2}} \omega u_{j}^{2} d x \\
& \quad+(3 \alpha-2) \int_{\mathbb{R}^{2}} \frac{h_{u_{j}}^{2}(|x|)}{|x|^{2}} u_{j}^{2} d x+\int_{\mathbb{R}^{2}} \lambda_{j} V\left(u_{j}^{2}\right) d x \\
& \quad-\alpha \int_{\mathbb{R}^{2}} \lambda_{j} V^{\prime}\left(u_{j}^{2}\right) u_{j}^{2} d x=0 .
\end{aligned}
$$

Then, from the change of variable and dividing by $T_{j}^{6 \alpha-4},(22)$ transforms to

$$
\begin{gathered}
\underbrace{\frac{\alpha}{T_{j}^{4 \alpha-4}} \int_{\mathbb{R}^{2}}\left|\nabla v_{j}\right|^{2} d x+\frac{\alpha-1}{T_{j}^{4 \alpha-2}} \int_{\mathbb{R}^{2}} \omega v_{j}^{2} d x+(3 \alpha-2) \int_{\mathbb{R}^{2}} \frac{h_{v_{j}}^{2}(|x|)}{|x|^{2}} v_{j}^{2} d x}_{A_{j}} \\
=\underbrace{\lambda_{j}\left[\frac{\alpha V^{\prime}\left(\left(T_{j}^{\alpha} v_{j}\right)^{2}\right)}{\left(\left(T_{j}^{\alpha} v_{j}\right)^{2}\right)^{2-1 / \alpha}}-\frac{V\left(\left(T_{j}^{\alpha} v_{j}\right)^{2}\right)}{\left(\left(T_{j}^{\alpha} v_{j}\right)^{2}\right)^{3-1 / \alpha}}\right]\left(v_{j}^{2}\right)^{3-1 / \alpha} d x .}_{B_{j}}
\end{gathered}
$$

Since $\left\|v_{j}\right\|=1$ and $T_{j}$ is unbounded, $A_{j}$ is bounded for $j$ but the structure assumption (V3) implies that $B_{j}$ tends to infinity as $j \rightarrow \infty$ provided $v_{0}$ is not identically zero. We claim that $v_{0}$ is nonzero. Suppose $v_{0}$ is identically zero. From (19) and (22), we see that

$$
\begin{aligned}
c_{u_{j}, \lambda_{j}}^{\prime}(1)= & \alpha \int_{\mathbb{R}^{2}}\left|\nabla u_{j}\right|^{2} d x+(\alpha-1) \int_{\mathbb{R}^{2}} \omega u_{j}^{2} d x \\
& +(3 \alpha-2) \int_{\mathbb{R}^{2}} \frac{h_{u_{j}}^{2}(|x|)}{|x|^{2}} u_{j}^{2} d x
\end{aligned}
$$




$$
\begin{aligned}
& +\int_{\mathbb{R}^{2}} \lambda_{j} V\left(u_{j}^{2}\right) d x \\
& -\alpha \int_{\mathbb{R}^{2}} \lambda_{j} V^{\prime}\left(u_{j}^{2}\right) u_{j}^{2} d x=0 .
\end{aligned}
$$

Then, Lemma 5 implies that $c_{u_{j}, \lambda_{j}}(1)$ is the global maximum of $c_{u_{j}, \lambda_{j}}(t)$ on $(0, \infty)$. Thus we see that, for each $R>1$,

$$
\begin{aligned}
C> & J_{\lambda_{j}}\left(u_{j}\right)=c_{u_{j}, \lambda_{j}}(1) \geq c_{u_{j}, \lambda_{j}}\left(R T_{j}^{-1}\right) \\
& =\frac{1}{2}\left(R^{2 \alpha} \int_{\mathbb{R}^{2}}\left|\nabla v_{j}\right|^{2} d x+R^{2 \alpha-2} \int_{\mathbb{R}^{2}} \omega v_{j} d x\right. \\
& +R^{6 \alpha-4} \int_{\mathbb{R}^{2}} \frac{h_{v_{j}}^{2}(|x|)}{|x|^{2}} v_{j}^{2} d x \\
& \left.-\int_{\mathbb{R}^{2}} \lambda_{j} V\left(\left(R^{\alpha} v_{j}(R x)\right)^{2}\right) d x\right) \geq \frac{1}{2} R^{2 \alpha-2}\left\|v_{j}\right\|^{2} \\
& -\frac{1}{2} \int_{\mathbb{R}^{2}} \lambda_{j} V\left(\left(R^{\alpha} v_{j}(R x)\right)^{2}\right) d x=\frac{1}{2} R^{2 \alpha-2}+o(1) .
\end{aligned}
$$

The last equality follows from $\left\|v_{j}\right\|=1$, the convergence of $\left\{v_{j}\right\}$ to 0 in $L^{q}\left(\mathbb{R}^{2}\right)$ for all $q>2$, and the structure conditions (V1)-(V2). However, taking large $R>1$, this makes a contradiction and shows $v_{0}$ is not identically zero. This proves the boundedness of $\left\{u_{j}\right\}$ in $H_{r}^{1}\left(\mathbb{R}^{2}\right)$.

Step 2 (compactness of $u_{j}$ ). Compactness of $\left\{u_{j}\right\}$ follows from a standard procedure. Since $\left\{u_{j}\right\}$ is bounded, there exists $u_{0} \in$ $H_{r}^{1}\left(\mathbb{R}^{2}\right)$ such that $\left\{u_{j}\right\}$ converges, up to a subsequence, to $u_{0}$ weakly in $H_{r}^{1}\left(\mathbb{R}^{2}\right)$ and strongly in $L^{q}\left(\mathbb{R}^{2}\right)$ for all $q>2$. Then it follows from Lemma 3 that $u_{0}$ is a critical point of $J$. Also, it is easy to see from the boundedness of $\left\{u_{j}\right\}$ that $J^{\prime}\left(u_{j}\right) \rightarrow 0$ in $H_{r}^{1}\left(\mathbb{R}^{2}\right)^{*}$. Recall that

$$
J(u)=\frac{1}{2}\|u\|^{2}+\widehat{J}(u)
$$

Using Lemma 3 once again, one can observe that

$$
\begin{aligned}
o(1)\left\|u_{j}\right\| & =J^{\prime}\left(u_{j}\right) u_{j}=\left\|u_{j}\right\|^{2}+\widehat{J}^{\prime}\left(u_{j}\right) u_{j} \\
& =\left\|u_{j}\right\|^{2}+\widehat{J}^{\prime}\left(u_{0}\right) u_{0}+o(1) \\
& =\left\|u_{j}\right\|^{2}-\left\|u_{0}\right\|^{2}+o(1)
\end{aligned}
$$

which shows $\left\|u_{j}\right\| \rightarrow\left\|u_{0}\right\|$ as $j \rightarrow \infty$. Therefore we have $u_{j} \rightarrow u_{0}$ in $H_{r}^{1}\left(\mathbb{R}^{2}\right)$ as $j \rightarrow \infty$.

\section{Construction of Approximate Solution Sequences}

In this section, we construct infinitely many approximate solution sequences. Choose an orthonormal basis $\left\{u_{i}\right\}_{i=1}^{\infty}$ of
$H_{r}^{1}\left(\mathbb{R}^{2}\right)$. For given $k \in \mathbb{N}$, let $Y_{k}$ and $Z_{k}$ be linear subspaces of $H_{r}^{1}\left(\mathbb{R}^{2}\right)$ spanned by $\left\{u_{1}, u_{2}, \ldots, u_{k}\right\}$ and $\left\{u_{k}, u_{k+1}, \ldots\right\}$, respectively. We will show that $J_{\lambda}$ enjoys a variant of symmetric mountain pass geometry (see [14]).

Lemma 8. There exist a sequence $\left\{k_{n}\right\} \in \mathbb{N}$ such that $k_{n} \rightarrow \infty$ as $n \rightarrow \infty$ and sequences $\left\{\rho_{n}\right\},\left\{r_{n}\right\} \in \mathbb{R}$ satisfying $\rho_{n}>r_{n}>0$ for each $n$ and

(i) $\inf \left\{J_{\lambda}(u) \mid u \in Z_{k_{n}}, T(u)=r_{n}\right\} \geq n$ for all $\lambda \in[1 / 2,1]$ and all $n$;

(ii) $\max \left\{J_{\lambda}(u) \mid u \in Y_{k_{n}}, T(u)=\rho_{n}\right\} \leq 0$ for all $\lambda \epsilon$ $[1 / 2,1]$ and all $n$.

Proof. We first show (i). The structure assumptions (V1)-(V2) imply that

$$
|V(s)| \leq \frac{\omega}{2}|s|+C|s|^{p}
$$

for some $C>0$ and all $s \geq 0$.

Then,

$$
\begin{aligned}
J_{\lambda}(u) \geq & \frac{1}{2} \int_{\mathbb{R}^{2}}|\nabla u|^{2} d x+\left(\frac{1}{2}-\frac{\lambda}{4}\right) \int_{\mathbb{R}^{2}} \omega u^{2} d x \\
& +\frac{1}{2} \int_{\mathbb{R}^{2}} \frac{h_{u}^{2}(|x|)}{|x|^{2}} u^{2} d x-\frac{C \lambda}{2} \int_{\mathbb{R}^{2}}|u|^{2 p} d x \\
\geq & \frac{1}{4} \int_{\mathbb{R}^{2}}|\nabla u|^{2} d x+\frac{1}{4} \int_{\mathbb{R}^{2}} \omega u^{2} d x \\
& -\frac{C}{2} \int_{\mathbb{R}^{2}}|u|^{2 p} d x .
\end{aligned}
$$

We recall the function $T$ and the linear isomorphism $L_{t}$ in Section 2. Let $v(x):=T(u)^{-\alpha} u\left(T(u)^{-1} x\right)$ so that $\|v\|=1$. By a change of variable, we get from (29) that, for each $u \in Z_{k}$,

$$
\begin{aligned}
J_{\lambda}(u) \geq & \frac{1}{4} T(u)^{2 \alpha} \int_{\mathbb{R}^{2}}|\nabla v|^{2} d x \\
& +\frac{1}{4} T(u)^{2 \alpha-2} \int_{\mathbb{R}^{2}} \omega v^{2} d x \\
& -\frac{C}{2} T(u)^{2 p \alpha-2} \int_{\mathbb{R}^{2}}|v|^{2 p} d x \\
\geq & \frac{1}{4} T(u)^{2 \alpha-2}-\frac{C}{2} T(u)^{2 p \alpha-2} \beta_{k, T(u)},
\end{aligned}
$$

where

$$
\begin{aligned}
\beta_{k, t} & :=\sup _{v \in W_{k, t}} \int_{\mathbb{R}^{3}}|v|^{2 p \alpha-2} d x, \\
W_{k, t} & =\left\{v \in H_{r}^{1}\left(\mathbb{R}^{2}\right) \mid\|v\|=1, v_{t}=t^{\alpha} v(t \cdot) \in Z_{k}\right\} .
\end{aligned}
$$

We claim that, for each $t>0, \beta_{k, t} \rightarrow 0$ as $k \rightarrow \infty$. To see this, suppose that $\beta_{k, t} \rightarrow \beta_{t}>0$ as $k \rightarrow \infty$. Choose $v_{k, t} \in W_{k, t}$ satisfying

$$
\int_{\mathbb{R}^{2}}\left|v_{k, t}\right|^{p} d x>\frac{\beta_{k, t}}{2} .
$$


Since $W_{k, t}$ is the unit sphere of a linear subspace $L_{t^{-1}}\left(Z_{k}\right)$ of $H_{r}^{1}\left(\mathbb{R}^{2}\right)$ with codimension $k-1$, we deduce $\left\{v_{k, t}\right\}$ converges to 0 weakly in $H_{r}^{1}\left(\mathbb{R}^{2}\right)$ and strongly in $L^{p}\left(\mathbb{R}^{2}\right)$, up to a subsequence. This however contradicts the fact that

$$
\lim _{k \rightarrow \infty} \int_{\mathbb{R}^{2}}\left|v_{k, t}\right|^{p} d x>\frac{\beta_{t}}{2}>0
$$

and the claim is true. We take $r_{n}=(8 n)^{1 /(2 \alpha-2)}$. Then, for any $u \in Z_{k}$ satisfying $T(u)=r_{n}$,

$$
J_{\lambda}(u) \geq 2 n-\frac{C}{2} r_{n}^{2 p \alpha-2} \beta_{k, r_{n}} .
$$

For each $n$, by taking sufficiently large $\left\{k_{n}\right\}$ satisfying

$$
\beta_{k_{n}, r_{n}}<\frac{2 n}{C r_{n}^{2 p \alpha-2}},
$$

we can see that the proof of (i) is complete.

Next we show (ii). Lemma 5 says that, for each $u \in$ $H_{r}^{1}\left(\mathbb{R}^{2}\right), J_{\lambda}\left(u_{t}\right) \rightarrow-\infty$ as $t \rightarrow \infty$. Also, we see from Lemma 6 and (20) that the set $\left\{u \in Y_{k} \mid T(u)=\rho\right\}$ is closed and bounded in finite dimensional space so it is compact. Combining these two facts with the compactness of $[1 / 2,1]$, we can deduce easily (ii) holds.

Define

$$
\begin{aligned}
& B_{n}:=\left\{u \in Y_{k_{n}} \mid T(u) \leq \rho_{n}\right\}, \\
& S_{n}:=\left\{u \in Z_{k_{n}} \mid T(u)=r_{n}\right\},
\end{aligned}
$$

with $k_{n}, \rho_{n}$, and $r_{n}$ given in Lemma 8 . Let $\Gamma_{n}$ be the set of continuous functions $\gamma: B_{n} \rightarrow H_{r}^{1}\left(\mathbb{R}^{2}\right)$ satisfying $\gamma(u)=u$ on $\partial B_{n}=\left\{u \in B_{n} \mid T(u)=\rho_{n}\right\}$. By $\gamma\left(B_{n}\right)$, we denote the set $\left\{\gamma(u) \in H_{r}^{1}\left(\mathbb{R}^{2}\right) \mid u \in B_{n}\right\}$.

Lemma 9 (intersection property). For any $n \in \mathbb{N}$, the intersection $\gamma\left(B_{n}\right) \cap S_{n} \neq \emptyset$ for every $\gamma \in \Gamma_{n}$.

Proof. Choose and fix arbitrary $n$ and $\gamma \in \Gamma_{n}$. Define

$$
U:=\left\{u \in \operatorname{int}\left(B_{n}\right) \mid T(\gamma(u))<r_{n}\right\},
$$

where $\operatorname{int}\left(B_{n}\right)$ denotes the interior of $B_{n}$ in $Y_{k_{n}}$. Then $U$ is a symmetric open neighborhood of 0 since $T$ is a continuous even map by Lemma 6 and $\gamma$ is a continuous odd map. Equation (20) in Lemma 6 says $B_{n}$ is bounded so that $U$ is also bounded. We claim that $T(\gamma(\partial U))=r_{n}$. From the continuity of $T$ and $\gamma$, it holds that $T(\gamma(\partial U)) \leq r_{n}$. Suppose that there is some $u_{0} \in \partial U$ such that $T\left(\gamma\left(u_{0}\right)\right)<r_{n}$. Then there is a neighborhood of $V$ of $u_{0}$ in $Y_{k_{n}}$ such that $T(\gamma(V))<r_{n}$. Choose some $v_{0} \in V \backslash U$. From the definition of $U, v_{0} \notin$ $\operatorname{int}\left(B_{n}\right)$. Since $v_{0} \in \partial U \subset B_{n}$, we see that $v_{0} \in \partial B_{n}$. Then, from the definition of $\gamma$, we have $T\left(\gamma\left(v_{0}\right)\right)=T\left(v_{0}\right)=\rho_{n}>r_{n}$, which is a contradiction. This shows the claim is true.

Now, consider a map $P_{n} \circ \gamma: \partial U \rightarrow Y_{k_{n}-1}$, where $P_{n}$ is the projection map from $H_{r}^{1}\left(\mathbb{R}^{2}\right) \rightarrow Y_{k_{n}-1}$. Then the well-known Borsuk-Ulam theorem applies to see the continuous odd map $P_{n} \circ \gamma$ has a vanishing point $u \in \partial U$; that is, $P_{n}(\gamma(u))=0$. This means that $\gamma(u) \in Z_{k_{n}}$. Therefore $\gamma(u) \in \gamma\left(B_{n}\right) \cap S_{n}$. The proof is complete.
Now, we are ready to prove the existence of infinitely many approximate solution sequences of $J$. For each $\lambda \epsilon$ $[1 / 2,1]$, we define infinitely many minimax levels as follows:

$$
C_{n}(\lambda):=\min _{\gamma \in \Gamma_{n}} \max _{u \in B_{n}} J_{\lambda}(\gamma(u))
$$

It follows from Lemmas 8 and 9 that $C_{n}(\lambda) \geq n$ for all $\lambda \epsilon$ $[1 / 2,1]$.

Proposition 10. For every fixed $n \in \mathbb{N}$, there exists an approximate solution sequence $\left\{\left(\lambda_{j}, u_{j}\right)\right\}$ of $J$ such that $J_{\lambda_{j}}\left(u_{j}\right)=$ $C_{n}\left(\lambda_{j}\right)$.

Proof. We invoke Theorem 2. From Lemmas 8 and 9, it holds that

$$
C_{n}(\lambda) \geq n>0 \geq \max _{v \in \partial B_{n}} J_{\lambda}(v)
$$

Let us check $J_{\lambda}$ enjoys property $(H)$. Let $\left\{\lambda_{j}\right\} \in[1 / 2,1]$ be a sequence strictly increasing to some $\lambda_{0} \in[1 / 2,1]$ and $\left\{v_{j}\right\} \in$ $H_{r}^{1}\left(\mathbb{R}^{2}\right)$ a sequence such that

$$
\begin{gathered}
-J_{\lambda_{0}}\left(v_{j}\right), \\
J_{\lambda_{j}}\left(v_{j}\right), \\
\frac{J_{\lambda_{j}}\left(v_{j}\right)-J_{\lambda_{0}}\left(v_{j}\right)}{\lambda_{0}-\lambda_{j}}<C .
\end{gathered}
$$

We need to show (i) $\left\{v_{j}\right\}$ is bounded in $H_{r}^{1}\left(\mathbb{R}^{2}\right)$ and (ii), for given $\varepsilon>0$, there exists $N>0$ satisfying

$$
J\left(\lambda_{0}, v_{j}\right) \leq J\left(\lambda_{j}, v_{j}\right)+\varepsilon \quad \forall n \geq N .
$$

We first show (i). We see from (40) that

$$
\begin{aligned}
& \frac{1}{2}\left\|v_{j}\right\|^{2}+\frac{1}{2} \int_{\mathbb{R}^{2}} \frac{h_{v_{j}}^{2}(|x|)}{|x|^{2}} v_{j}^{2} d x<2 C, \\
& -C<\frac{1}{2} \int_{\mathbb{R}^{2}} V\left(u_{j}^{2}\right) d x<C,
\end{aligned}
$$

which shows $\left\{v_{j}\right\}$ is bounded in $H_{r}^{1}\left(\mathbb{R}^{2}\right)$. Also, for given $\varepsilon>0$,

$$
\begin{aligned}
J\left(\lambda_{0}, v_{j}\right)-J\left(\lambda_{j}, v_{j}\right) & =\left(\lambda_{j}-\lambda_{0}\right) \frac{1}{2} \int_{\mathbb{R}^{2}} V\left(u_{j}^{2}\right) d x \\
& \leq\left(\lambda_{0}-\lambda_{j}\right) C \leq \varepsilon
\end{aligned}
$$

if $j$ is sufficiently large. This shows (ii). Therefore, there exists a subset $M \subset[1 / 2,1]$ with full measure in $[1 / 2,1]$ that, for every $\lambda \in M$, there exists a bounded (PS) sequence $\left\{u_{\lambda, k}\right\}_{k=1}^{\infty}$ of $J_{\lambda}$ at level $C_{n}(\lambda)$. Arguing similarly to Step 2 of Proposition 7, we also deduce $\left\{u_{\lambda, k}\right\}_{k=1}^{\infty}$ converges, up to a subsequence, to some critical point $u_{\lambda}$ of $J_{\lambda}$ with $J_{\lambda}\left(u_{\lambda}\right)=$ $C_{n}(\lambda)$. Since $M$ has full measure in $[1 / 2,1]$, this completes the proof. 
Completion of the Proof of Theorem 1. Now we complete the proof of Theorem 1 . We first choose and fix arbitrary $n \in \mathbb{N}$. Let $\left\{u_{j}\right\}$ be an approximate solution sequence of $J_{\lambda_{j}}$, given by Proposition 10. Take $\gamma \in \Gamma_{n}$ satisfying

$$
\max _{u \in B_{n}} J(\gamma(u)) \leq C_{n}(1)+\frac{1}{2} .
$$

It follows from the compactness of $B_{n}$ that

$$
\begin{aligned}
C_{n}\left(\lambda_{j}\right) \leq & \max _{u \in B_{n}} J_{\lambda_{j}}(\gamma(u)) \\
\leq & \max _{u \in B_{n}} J(\gamma(u)) \\
& +\left(1-\lambda_{j}\right) \max _{u \in B_{n}} \int_{\mathbb{R}^{2}} \frac{1}{2} V\left(\gamma(u)^{2}\right) d x \\
\leq & C_{n}(1)+\frac{1}{2}+\frac{1}{2}=C_{n}(1)+1
\end{aligned}
$$

for sufficiently large $j$. Then Proposition 7 applies to see $u_{j}$ converges, up to a subsequence, to some $u_{0}$ which is a critical point of $J$. Recall that $J_{\lambda_{j}}\left(u_{j}\right)=C_{n}\left(\lambda_{j}\right) \geq n$. By taking a limit $j \rightarrow \infty$, we deduce $J\left(u_{0}\right) \geq n$. Since $n$ is arbitrary, this shows the existence of infinitely many critical points of $J$. This completes the proof.

\section{Conflict of Interests}

The author declares that there is no conflict of interests regarding the publication of this paper.

\section{Acknowledgments}

The author was supported by Basic Science Research Program through the National Research Foundation of Korea (NRF) funded by the Ministry of Education (NRF2014R1A1A2054805) and also was supported by the POSCO TJ Park Science Fellowship.

\section{References}

[1] R. Jackiw and S.-Y. Pi, "Soliton solutions to the gauged nonlinear Schrödinger equation on the plane," Physical Review Letters, vol. 64, no. 25, pp. 2969-2972, 1990.

[2] R. Jackiw and S.-Y. Pi, "Classical and quantal nonrelativistic Chern-Simons theory," Physical Review D, vol. 42, no. 10, pp. 3500-3513, 1990.

[3] J. Byeon, H. Huh, and J. Seok, "Standing waves of nonlinear Schrödinger equations with the gauge field," Journal of Functional Analysis, vol. 263, no. 6, pp. 1575-1608, 2012.

[4] A. Pomponio and D. Ruiz, "A variational analysis of a gauged nonlinear Schrödinger equation," Journal of the European Mathematical Society, vol. 17, no. 6, pp. 1463-1486, 2015.

[5] A. Pomponio and D. Ruiz, "Boundary concentration of a Gauged nonlinear Schrödinger equation on large balls," Calculus of Variations and Partial Differential Equations, vol. 53, no. 1-2, pp. 289-316, 2015.

[6] H. Huh, "Standing waves of the Schrödinger equation coupled with the Chern-Simons gauge field," Journal of Mathematical Physics, vol. 53, no. 6, Article ID 063702, 2012.
[7] A. Ambrosetti and P. H. Rabinowitz, "Dual variational methods in critical point theory and applications," Journal of Functional Analysis, vol. 14, no. 4, pp. 349-381, 1973.

[8] P. Cunha, P. d'Avenia, A. Pomponio, and G. Siciliano, "A multiplicity result for Chern-Simons-Schrödinger equation with a general nonlinearity," Nonlinear Differential Equations and Applications, In press.

[9] H. Berestycki and P.-L. Lions, "Nonlinear scalar field equations. I. Existence of a ground state," Archive for Rational Mechanics and Analysis, vol. 82, no. 4, pp. 313-345, 1983.

[10] J. Tan and Y. Wan, "Standing waves for the Chern-SimonsSchrödinger systems without (AR) condition," Journal of Mathematical Analysis and Applications, vol. 415, no. 1, pp. 422-434, 2014.

[11] J. Seok, "On nonlinear Schrödinger-Poisson equations with general potentials," Journal of Mathematical Analysis and Applications, vol. 401, no. 2, pp. 672-681, 2013.

[12] M. Struwe, "The existence of surfaces of constant mean curvature with free boundaries," Acta Mathematica, vol. 160, no. 1, pp. 19-64, 1988.

[13] L. Jeanjean and J. F. Toland, "Bounded Palais-Smale mountainpass sequences," Comptes Rendus de l'Academie des SciencesSeries I: Mathematics, vol. 327, no. 1, pp. 23-28, 1998.

[14] M. Willem, Minimax Theorems, vol. 24 of Progress in Nonlinear Differential Equations and Their Applications, Birkhäuser, Boston, Mass, USA, 1996. 


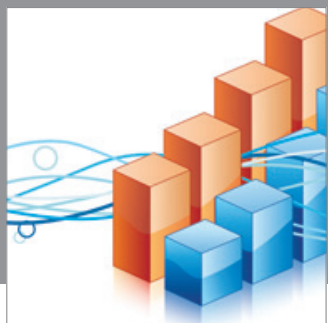

Advances in

Operations Research

mansans

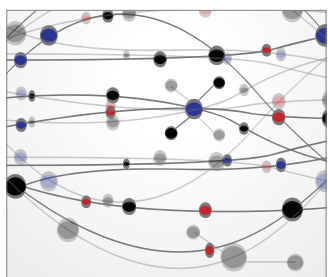

The Scientific World Journal
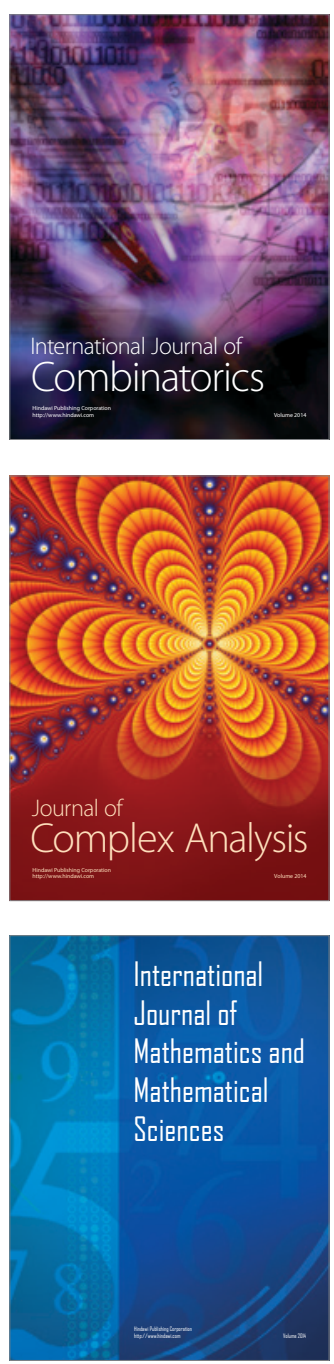
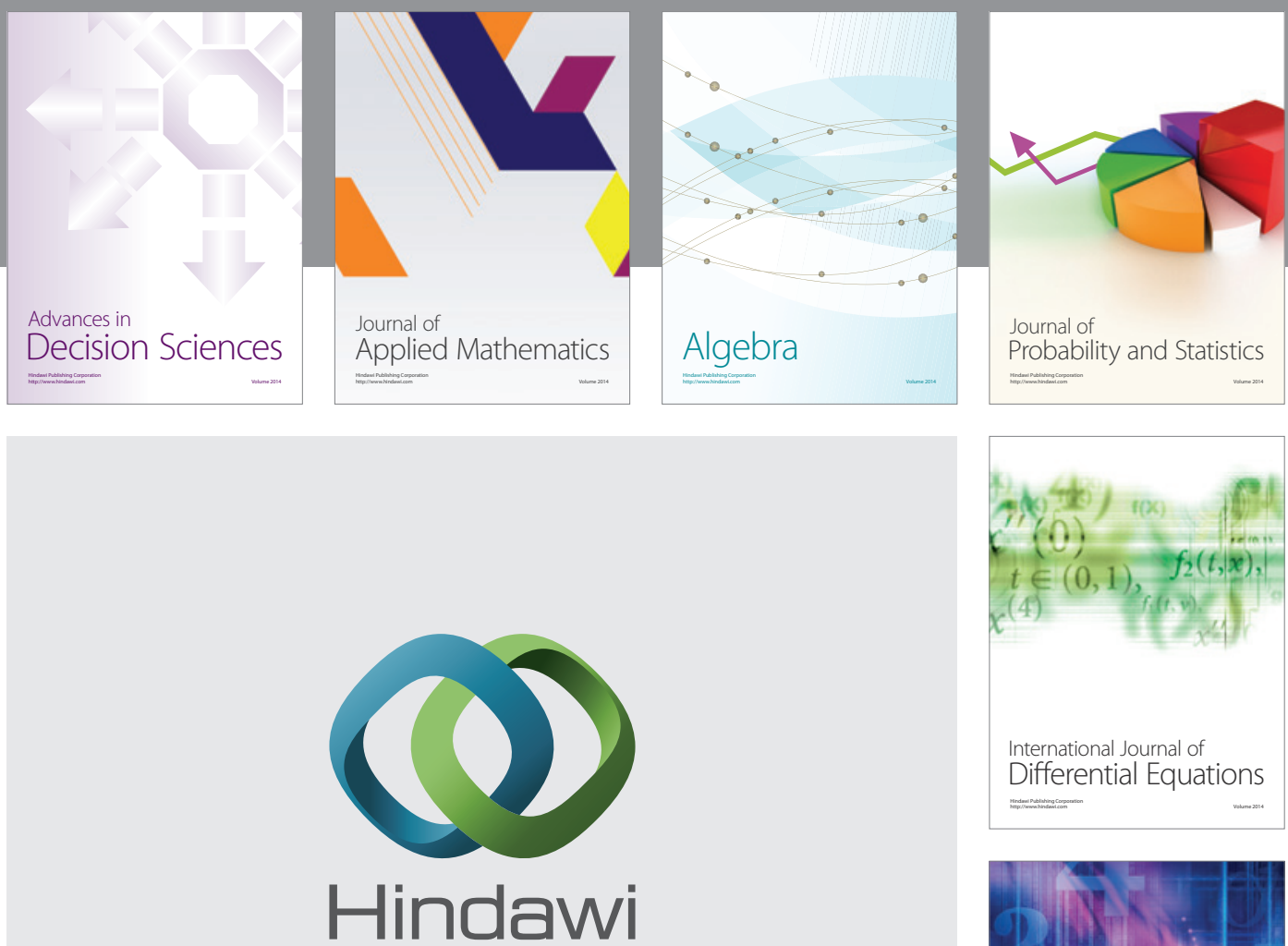

Submit your manuscripts at http://www.hindawi.com
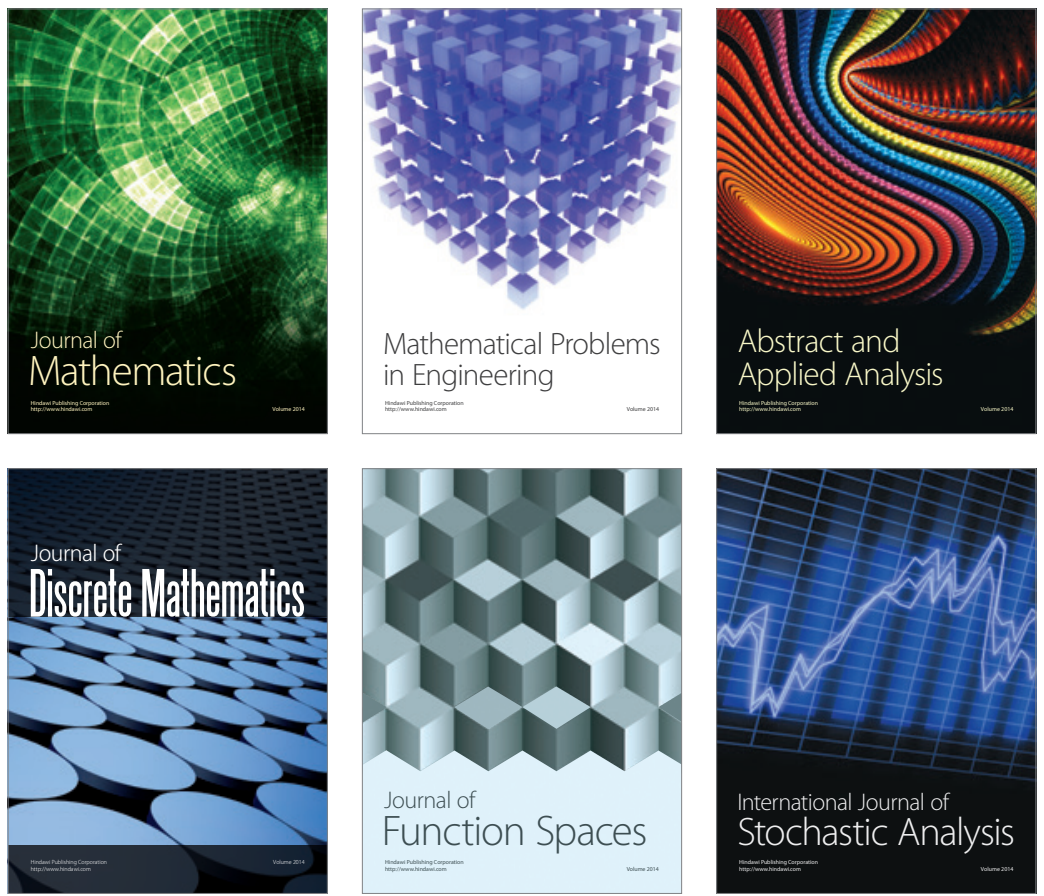

Journal of

Function Spaces

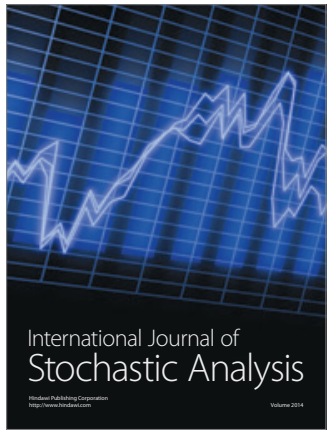

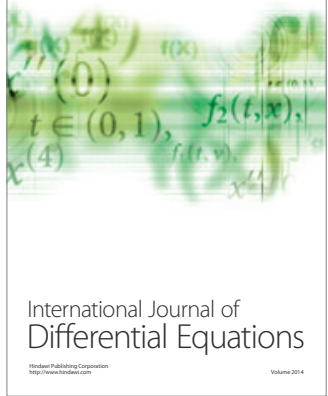
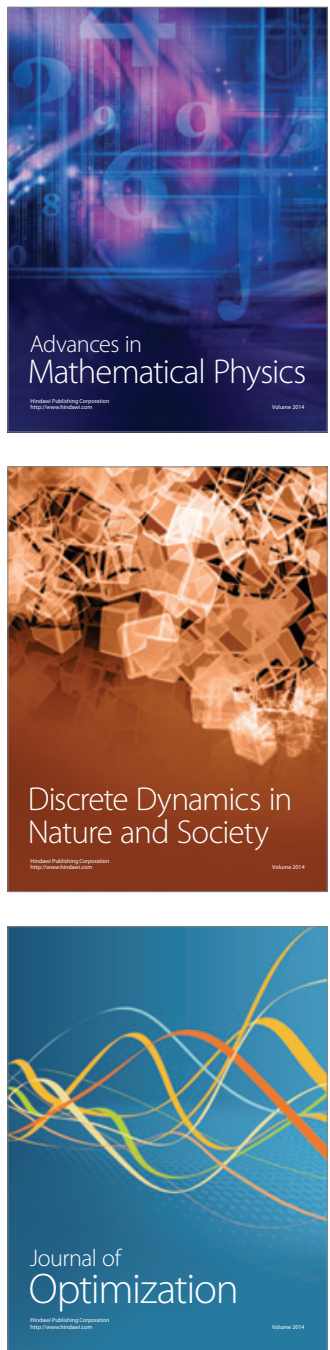